\title{
Albertine dans "Le Rideau cramoisi"
}

Catherine PARAYRE

T e mystère est certainement un des termes qui viennent de suite à l'esprit à l'évocation de l'énigmatique Albertine dans "Le Rideau cramoisi" (Petit 129, Kelly 141, Berthier, Une écriture du désir 106). En effet, les agissements et les motivations de la jeune fille semblent dissimulés et difficilement compréhensibles. Le narrateur principal est précisément captivé par le récit du vieux Brassard parce que, depuis sa première apparition dans la salle à manger jusqu'à sa mort inattendue, Albertine est un personnage entouré d'un grand mystère. Le rideau cramoisi que le narrateur et Brassard observent, assis dans la diligence, en est le symbole: le rideau cache une scène à l'intérieur de la demeure alors que la lumière tamisée la filtre. Le rideau cramoisi, par l'atmosphère qu'il évoque dans la nuit, manifeste ce mystère qui enveloppe le récit de Brassard. Toutefois, par bien des signes et des détails dans la narration, il s'avère possible de qualifier ce mystère, d'affiner cette vision d'Albertine et de parvenir à déceler en elle un code, une cohérence interne qui permettent en quelque sorte de décrypter le 
personnage. En effet, Brassard, en tant que narrateur et personnage majeur du récit, va bien au-delà de la simple notion de mystère. Il l'approfondit et la lit différemment; il lui donne une profondeur toute codifiée et organisée. Il interprète le personnage d'Albertine à sa façon et lui confère tout au long de son récit une signification et des structures. Le récit de Brassard abonde aussi en codes divers qui, d'une certaine manière, expliquent le personnage de la jeune fille et diluent progressivement l'impression de mystère pour laisser place au portrait extrêmement bien régi et organisé d'une jeune fille extraordinaire. Albertine est mystérieuse, mais surtout elle est hors du commun, hors des normes, et tout dans son portrait s'assemble pour en donner cette impression. Le discours du mystère n'est en fait qu'un affleurement; Albertine est bel et bien un être hyperbolique dont la création est parfaitement codifiée. Ceci est mis en évidence par une série d'éléments récurrents dans la nouvelle: le discours hyperbolique qu'emploie Brassard pour faire part de ses impressions, les images de métamorphose qui se retrouvent à intervalles réguliers au cours de la narration, les réseaux d'images organisés de telle manière qu'ils s'articulent sur de grands axes d'opposition, et finalement le thème du dandysme chez Albertine qui se développe tout au long du récit. Ces différents aspects présents dans la narration de Brassard effacent peu à peu le mystère et indiquent en une trame serrée et rigoureusement codifiée ce qui est extraordinaire chez Albertine.

Le premier procédé de codification qui apparaît est l'usage répété de l'hyperbole dans la narration de Brassard. Albertine est de suite perçue comme un être grandi, magnifié. En fait, dès sa première apparition, Albertine est qualifiée en termes d'exagération: "cambrée à outrance" (30), elle, montre une taille prise "(c'est le mot tant elle était lacée)" (30) dans un corset qui met en valeur sa finesse. Ainsi, Albertine est de suite caractérisée emphatiquement. Même sa posture est grandiose: "debout et sur la pointe des pieds" (30), elle s'offre en spectacle. Les procédés hyperboliques pour la décrire s'accumulent dès lors. 
Les procédés d'intensification se retrouvent régulièrement dans la narration. Par exemple, la politesse d'Albertine est "la politesse la plus froide" (32); sa passion est "un despotisme si insensément passionné" (33); son regard est une "si monstrueuse avance "(35); la jeune fille est "si diaboliquement provocante" (36) et cette provocation se fait avec "une telle prestesse" (37), "si sûre d'ellemême" (38). En revanche, "elle n'avait rien de ces peurs vulgaires et osées" (45). Cette série de clauses introduites par le mot "si" ou un mot similaire contribuent à ajouter à l'outrance qui apparaît dès le début de la narration par Brassard.

A ce procédé s'associe également le choix des termes utilisés qui font souvent référence à des situations superlatives: Brassard "[crible Albertine] d'épithètes" (40) qui dénotent "l'outrance où elle [1]'avait jeté" (40). En effet, Albertine n'est pas simplement aguichante et tentatrice; elle l'est "diaboliquement" (36). Ou encore sa propensité au mal n'est pas uniquement précoce, elle l'est d'une manière "effrayante" (35).

En fait, toute la narration que fait Brassard de cet épisode de sa jeunesse est caractérisée par de tels procédés, comme si le récit était exagéré à souhait, rendu superlatif. Il n'est pas de paragraphe qui soit simple ou sobre. Brassard est prolixe et bavard. D'ailleurs, le narrateur de la nouvelle explique: "Il avait le propos vif. Le capitaine de Brassard allait toujours trop loin" (17). Son récit est donc caractérisé par une outrance dans le propos. Chaque fois que Brassard cherche à qualifier le personnage d'Albertine, il est amené à le faire à l'excès.

L'excès est aussi le propre d'un autre procédé qui apparaît de façon significative et récurrente dans la narration et qui concerne les phénomènes de transformation qui affectent Albertine. En effet, le récit que Brassard fait de son idylle avec la jeune fille est jalonné par une série d'images métamorphiques. Le terme de métamorphose s'applique ici dans la mesure où il $\mathrm{y}$ a une transformation, un changement d'une nature à une autre, d'un état à un autre. Tout d'un coup, Albertine, fille d'un couple bourgeois d'une petite ville de 
province, se métamorphose en une série de personnages imaginaires. La description d'Albertine répond à un code précis et élaboré qui s'articule sur le thème de l'extraordinaire.

Dès son entrée dans la salle à manger lors de leur première rencontre, Brassard est frappé par la pose de la jeune fille: Albertine est d'emblée une danseuse. L'image est féminine, mais aussi quasi immobile puisque la jeune fille ne bouge que "lentement" (30), et froide, la danse évoquant non pas un monde aux couleurs chatoyantes mais, au contraire, un monde bien organisé et très sobre.

Albertine devient ensuite une reine ou une princesse: "L'Infante à l'épagneul de Velasquez, pourrait ... vous donner une idée de cet air-là" (31). Cette image de métamorphose en princesse est d'ailleurs continuée à plusieurs reprises dans la narration. En effet, Albertine est "cette archiduchesse d'altitude" (32) "à l'air si déplacé d'Infante" (33), qui agit avec le jeune garçon avec "despotisme" (33) et "souveraineté" (34). Albertine est "la grande Mademoiselle Impassible" (41), "toujours la Madame Infante" (48) avec "son air de Princesse de cérémonie" (35).

Un peu plus tard, Albertine est comparée à Niobé, princesse qui, pour protéger ses enfants, fut métamorphosée en pierre. Autre métamorphose, celle d'Albertine en diablesse. Cette fois, Brassard dessine la jeune fille avec "le visage de cette diablesse de femme dont [il] était possédé, comme les dévôts disent qu'on l'est du diable" (43). Finalement, Albertine devient sphinx, un de "ces animaux fabuleux" (47), et "paraissait plus Sphinx à elle seule, que tous les Sphinx dont l'image se multipliait ... dans cet appartement Empire" (47).

Ainsi, ces images qui évoquent toutes plusieurs degrés de métamorphoses et plusieurs degrés de parenté entre elles forment un réseau métaphorique qui contribue à qualifier le personnage d'Albertine: Albertine éveille l'étonnement, l'émerveillement. Chaque fois, elle apparaît comme un être supérieur, potentiellement dangereux pour Brassard. Elle est donc caractérisée par des traits d'une féminité redoutable et hors du commun, hors des 
normes. Le procédé utilisé par Brassard pour dépeindre Albertine - l'utilisation répétée d'images métamorphiques - permet de mettre en relief les caractéristiques superlatives du personnage.

Cette Albertine extraordinaire est également décrite en termes d'oppositions. Le premier réseau d'images à apparaître est celui qui oppose la danseuse, personnage quasi angélique, gracieux et éthéré, "tombée du ciel" (32) avec l'évocation diabolique qui grandit progressivement au cours de la narration. Bien qu'elle ait "une aisance et une gracieuse langueur de mouvements incomparables" (34), la jeune fille devient "diaboliquement provocante" (36). "Cette Albertine d'enfer" (42) est une "diablesse" (43) et son apparence physique lors de ses rencontres nocturnes avec Brassard est particulièrement évocatrice: "les yeux tout grands, - des yeux immenses! - . . à la noirceur profonde" (45), un teint "horriblement pâle" et des "lèvres rouges et érectiles" (45). Albertine est tout à la fois divine et diabolique.

Ce système d'opposition s'étoffe avec la contradiction latente de la folie et de la sobriété. En effet, Albertine a les gestes contenus et mesurés d'une danseuse; elle est posée et tranquille, calme, délicate et raffinée. C'est "une jeune fille bien élevée, sans affectation, habituellement silencieuse, qui, quand elle parlait, disait en bons termes ce qu'elle avait à dire, mais qui n'outrepassait jamais cette ligne-là" (32). Albertine est "sobre" (32), un être caractérisé par la simplicité et un "ton dégagé' (39). Toutefois, son comportement trahit aussi "l'égarement" (34). En effet, sur son visage, si pâle et expressif, Brassard voit "passer de la démence" (45). Ici aussi, la description s'étale d'un antipode à l'autre. Albertine est à la fois maîtresse de ses actions et folle.

Sa personnalité est également divisée entre le calme et la peur. Très sûre d'elle, elle rend visite à Brassard durant la nuit, n'hésitant pas à traverser la chambre de ses parents endormis. Elle est alors pleine d'un "sang-froid" (34) qui impressionne fortement le jeune Brassard. Elle n'a aucune peine à "s'expos(er) de plus belle au danger bravé" (49). Rien ne semble l'arrêter dans sa détermina- 
tion à agir à sa guise. Albertine étonne Brassard par son courage, qui peut presque être qualifié de témérité car elle ne connaît "rien de ses peurs vulgaires" (45). Pourtant, selon Brassard, "sa peur . . . devait être immense" (44). Elle connaissait certainement "l'effroi" (44) et la terreur. Son comportement quand elle se jette, éperdue, dans les bras de Brassard, peut être interprété comme de la peur et de la faiblesse. Ici encore, les traits significatifs du personnage s'articulent sur une franche opposition.

Un autre paradoxe se retrouve dans l'opposition de l'impassibilité et de la ferveur qui, simultanément, caractérisent la jeune fille et qui sont le plus souvent évoquées par les images du froid et du chaud. Ses yeux sont "glacés" (39), muets et impassibles alors que sa présence fait "bouillir" (38) Brassard de curiosité et autres sentiments confus. Son visage blanc et égaré évoque la froideur alors qu'elle donne à Brassard un "baiser de feu" (45).

La dernière opposition élaborée dans la narration est celle du féminin et du masculin. Albertine, personnage absolument féminin, est aussi caractérisée en termes masculins (Sivert 153). Par exemple, sa main est "un peu grande et forte comme celle d'un jeune garçon" (Barbey d'Aurevilly 33), "un peu épaisse" (34). Surtout, sa délicatesse de jeune fille de bonne famille contraste avec son audace dans ses avances à Brassard. C'est elle qui le provoque et prend sa main sous la table. C'est elle qui mène l'affaire, d'une façon bien plus masculine que le jeune Brassard qui se laisse aller au gré des désirs de la jeune fille. Albertine est décidée, volontaire comme un homme. C'est elle qui déclare en fait ses sentiments à Brassard, et non le contraire.

Cette série d'oppositions met donc en avant la complexité du personnage. Albertine est un personnage déconcertant, contradictoire, riche et compliqué, en fait un être hors du commun. Sa description correspond à un code précis et clair. La série d'oppositions qui peut y être décelée indique combien le personnage fait montre d'une remarquable ampleur. 
Albertine, dont la description parvient au lecteur à travers le regard et le point de vue de Brassard, est également évoquée en termes qui correspondent à la philosophie de ce dernier. Cette transition du regard est mise en évidence dans la longue introduction qui présente Brassard dans ses moindres détails. Le narrateur y indique ses traits de caractère ainsi que les faits significatifs de sa carrière. Cette partie de l'histoire, qui sert de cadre à la narration de Brassard lui-même, constitue un moment crucial dans la narration: "Si le capitaine vicomte de Brassard n'avait pas été tout ce que je viens d'avoir l'honneur de vous dire, mon histoire serait moins piquante, et probablement n'eussé-je pas pensé à vous la conter" (16). C'est pour dire que la description, non pas d'Albertine, mais de Brassard est importante. Or, cette description fait apparaitre Brassard tout d'abord comme un jeune dandy novice et peu expérimenté, mais très décidé, et progressivement comme un dandy vétéran et confirmé.

C'est cette éthique du dandysme qui se reporte du personnage de Brassard sur le personnage d'Albertine. Albertine se comporte en fait comme un dandy (Berthier 93, Sivert 154). Brassard voit en elle un personnage qui lui ressemble presque identiquement.

Tout d'abord, Albertine se donne en spectacle. Or, le dandy, comme Brassard dans sa jeunesse quand il se rend à l'émeute "en chaussons vernis et en bas de soie" (15), est un être qui se fait remarquer, auquel il faut des spectateurs, et qui provoque le regard d'autrui. Voici ce qu'écrit de Liedekerke dans Talon Rouge: "L'obsession de se dédouaner du monde, de faire cavalier seul, se double du désir d'être reconnu. Le dandy est un acteur. Il lui faut un parterre, un public" (86). Boschian-Campaner ajoute dans son livre Barbey d'Aurevilly: "Le dandy a besoin de spectateurs, il lui faut une scène" (80). C'est bien ce qui se passe avec Albertine. Dès la première scène, elle est une danseuse, dans la position de la ballerine, et elle s'offre au regard admiratif du jeune Brassard.

Un autre trait qu'il révèle et amplifie chez Albertine et qui est un trait significatif du dandysme, est son arrogance. En effet, l'impertinence, "fille de la Légèreté et de 1'Aplomb" selon Barbey 
d'Aurevilly (Sur le Dandysme 138), est un signe majeur du dandysme. Il correspond à la fois à l'aisance et au mépris du conformisme et de la routine. Albertine se comporte précisément ainsi. Lorsqu'elle apparaît telle une danseuse dans la salle dans laquelle Brassard vient de pénétrer, elle accroche son regard par la lenteur et l'indifférence de ses gestes, qu'elle accomplit "presque impertinemment" (Barbey d'Aurevilly, Les Diaboliques 30). Albertine est une "calme et insolente fille" (33). "Etait-elle effrontée ?"(34) se demande Brassard lorsqu'il découvre son attitude et ses manières. En effet, Albertine se comporte avec "cette impudeur et: cette impudence" (36) qui choquent le jeune soldat. Albertine ne se soucie guère des bienséances. Elle est arrogante dans ses gestes aisés et gracieux, mais aussi dans ses actions lorsqu'elle séduit Brassard, puis, plus tard, quand elle s'introduit dans sa chambre malgré tous les risques.

Elle est surtout un être paradoxal et contradictoire, ce qui la rend véritablement dandy et, bien sûr, infiniment intéressante. Elle évoque un personnage de dandy parce que, comme le démontre les images métamorphiques qui parsèment le récit, elle est un être supérieur et dangereux. En outre, ses attitudes sont dictées par des motifs qui s'opposent; elle est ainsi décrite en termes contradictoires dans des réseaux qui se développent tout au long de la narration.

Ce paradoxe inné éveille chez Brassard toute une gamme de sentiments qui s'étalent de la simple curiosité à une stupéfaction très forte. Les références à ces sentiments sont particulièrement fréquentes. Ainsi, dès le premier regard qu'il jette sur la jeune fille, son "étonnement de la voir là fut de beaucoup dépassé par l'éton-" nement de savoir qui elle était" (31). Il est "encore plus surpris" (35) par ce qui suit: "ceci déconcertait mon petit aplomb de: Lovelace de dix-sept ans" (35), dit-il, "ceci me paraissait plus fort que ce que j'avais lu" (35). Albertine éveille en lui des sentiments "de curiosité, de contrariété, d'inquiétude, d'un tas de sentiments agités et déçus" (38). Cette recherche de l'étonnement et de la sur-" prise a des implications très étendues car, comme l'explique Barbey 
d'Aurevilly dans son traité Sur le dandysme, il vaut mieux "étonner que plaire ... . le plus beau des étonnements, c'est l'épouvante" (136). En effet, Brassard arrive à ce paroxysme de sentiments à la mort d'Albertine qui ne cesse donc jamais de le surprendre ni de le stupéfier. Voici ce qu'explique Brassard en introduction au récit de la mort d'Albertine:

Elle m'avait passé dans l'âme plus d'un genre de frisson, plus d'un genre de terreur ... Eh bien ce fut plus que cela. Ce fut de la peur, de la peur complète, de la vraie peur . . . ; ce fut cette panique qui fait prendre la fuite à des régiments tout entiers" (50).

Albertine est donc un personnage de dandy particulièrement réussi car elle parvient à la perfection à éveiller ces émotions-là chez Brassard.

Tous les paradoxes qui l'habitent, toute sa dangerosité féminine culminant dans sa mort, font d'elle un personnage auquel s'appliquent tous les qualificatifs du dandysme. Elle est "extraordinaire en tout" (Barbey d'Aurevilly, dandysme 171). Brassard dresse donc le portrait d'un dandy féminin. Tout en elle, l'outrance dans la description, les paradoxes qui s'établissent à son égard, la supériorité effrayante qui se manifeste, peut être réduit à cette esthétique du dandy. Albertine est donc un personnage non pas tant mystérieux que codifié à l'extrême.

University of Kansas 


\section{Ouvrages cités}

Balzac, Baudelaire, Barbey d'Aurevilly. Sur le dandysme. Paris: 10/18, 1971.

Barbey d'Aurevilly, Jules. Oeuvres romanesques complètes. Paris: Gallimard (Pléiade), 1966.

Berthier, Philippe, ed. Barbey d'Aurevilly cent ans après. Geneva: Droz, 1990.

---. L'Ensorcelée - Les Diaboliques de Barbey d'Aurevilly. Une écriture du désir. Paris: Champion, 1987.

Boschian-Campaner, Catherine. Barbey d'Aurevilly. Paris: Librairie Séguier, 1989.

Kelly, Dorothy. "Seeing Albertine Seeing: Barbey and Proust through Balzac." Studies in Twentieth Century Literature 14.2 (Summer 1990): 139-57.

Liedekerke, Amould de. Talon Rouge, Barbey d'Aurevilly, le dandy absolu. Paris: Olivier Orban, 1986.

Petit, Jacques. "La femme dominatrice." Revue des Lettres Modernes 351-54 (1973)

Sivert, Eileen. "Narration and Exhibitionism in 'Le Rideau cramoisi'." Romanic Review 70 (1979): 146-58. 

